\title{
Prognostic Value of PRISMA-7 Score for Frail Elderly Patients with Advanced Pancreatic Adenocarcinoma
}

ISSN: 2637-773X

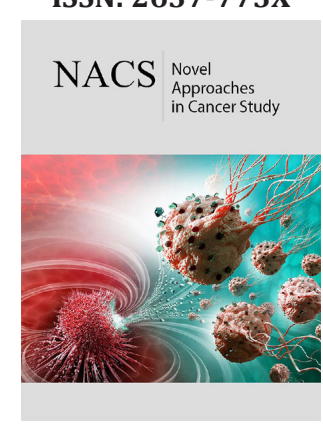

*Corresponding authors: Salah Mabrouk Khallaf, Medical Oncology Lecturer, Assiut, Egypt

Submission: 眥 February 27, 2020

Published: 漹October 11, 2021

Volume 6 - Issue 3

How to cite this article: Salah Mabrouk Khallaf*, Anwar Tawfik Amin, and Mona M Sayed. Prognostic Value of PRISMA-7 Score for Frail Elderly Patients with Advanced Pancreatic Adenocarcinoma. Nov Appro in Can Study. 6(3). NACS.000638. 2021. DOI: 10.31031/NACS.2021.06.000638

Copyright@ Salah Mabrouk Khallaf, This article is distributed under the terms of the Creative Commons Attribution 4.0 International License, which permits unrestricted use and redistribution provided that the original author and source are credited.

\author{
Salah Mabrouk Khallaf ${ }^{1 *}$, Anwar Tawfik Amin², and Mona M Sayed ${ }^{3}$ \\ ${ }^{1}$ Medical Oncology Department, Egypt \\ ${ }^{2}$ Surgical Oncology Department, Egypt \\ ${ }^{3}$ Radiation Oncology Department, Egypt
}

\begin{abstract}
Finding the prognostic factors for this dismal disease is still challenging, especially for elderly patients. Patient age [1,2], performance status [1], blood neutrophil-lymphocyte ratio [3], Plasma Chromogranin A [4], frailty index [5] are documented as prognostic factors for outcome of the patients with advanced pancreatic cancer. Data about elderly patients in these trials is limited. We present our practice regarding the importance of frailty index called PRISMA7 (Program of Research to Integrate the Services for the Maintenance of Autonomy-7) score for prognostic stratification of elderly patients with advanced pancreatic cancer. It was highly significant prognostic factor for those patients.
\end{abstract}

Keywords: Cancer pancreas; PRIMSA7; Frail; Prognosis

Abbreviations: CGA: Comprehensive Geriatric Assessment; PRISMA-7: Program of Research to Integrate the Services for the Maintenance of Autonomy-; ECOG: Eastern Cooperative Oncology Group; PFS: Progression-Free Survival; OS: Overall Survival

\section{Discussion}

Pancreatic cancer is a major health problem worldwide ranking as the 11th most common cancer, and the 7th most common cause of death from cancer worldwide [6]. Its worldwide incidence and mortality increase with increasing age [6]. About two-thirds of patients have the age of more than 65 years [7-9]. The age of 65 years is the cut-off for the definition of the elderly according to the proposal of the World Health Organization [10]. In cancer clinical trials, data about this age group is inadequate as the number of patients of this age group accounts for only $34-40 \%$ of enrolled patients [11]. Because of the biophysiological nature of the elderly patients, age and performance status are not preferred for clinical judgment and clinical studies1 [12,13]. Alternatively, a Comprehensive Geriatric Assessment (CGA) is used as a reliable prognostic factor for survivals [14]. It also a good predictive model for chemotherapy toxicity [14]. The clinical application of CGA is restricted by its time and resource-consumption [8]. So, many CGA-based frailty indices have emerged for clinical application [15].

Among these indices, the PRISMA-7 (Program of Research to Integrate the Services for the Maintenance of Autonomy-7) scale is an applicable, valid, and reliable tool for research and clinical practice [15]. PRISMA-7 is self-reported scale and contains seven items of 'yes/ no' answer: older than 85 years; male; health problems which limit activities; support of another person needed on a regular basis; health problems requiring staying at home; social support; and use of a stick/walker/wheelchair [16]. Each item is scored with one point for a "yes" answer, with a total score $\geq 3$ indicates frailty [16]. In our practice, we conducted a 
trial is to evaluate the prognostic factors of metastatic pancreatic adenocarcinoma in the frail elderly patients treated with gemcitabine in a standard dose. The eligibility criteria were the evidence of the diagnosis of metastatic pancreatic adenocarcinoma, both sexes, age of 65 years or older, PRISMA-7 score $\geq 3$ points, an Eastern Cooperative Oncology Group (ECOG) performance status 2, chemotherapy-naïve, and adequate hematologic, hepatic, and renal functions. Also, palliative radiotherapy for distant metastasis was allowed if indicated.

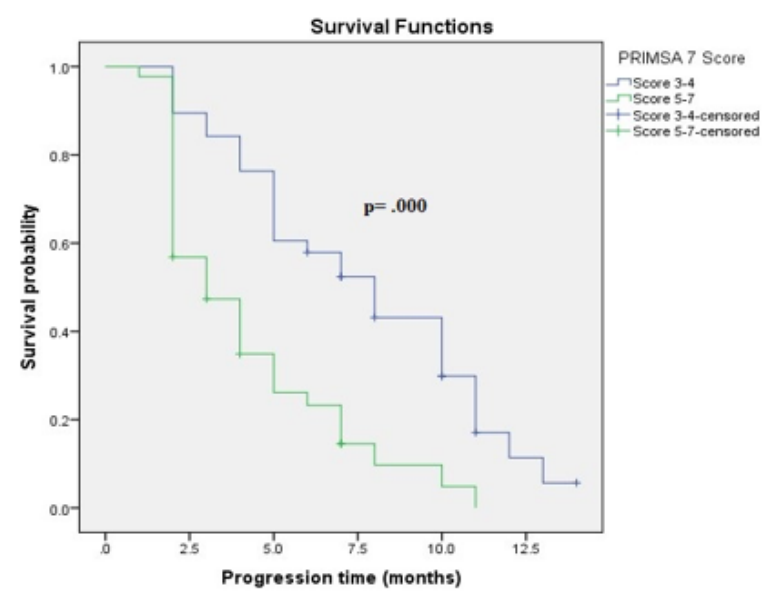

Figure 1: Kaplan-Meier plot progression-free survival according to PRISMA7 score.

During the study period from May 2016 to September 2019, eighty-two patients out of the assessed patients were finally analyzed for study endpoints. We subclassified the frail patients according to the PRISMA7 score. A total of score of 3 or 4 was considered mildly frailty, while the moderately to severely frail patients had a total score of 5-7. We found that the patients having high PRISMA7 score (moderately to severely frail patients) had a worse Progression-Free Survival (PFS) than those with low score i.e., mildly frail patients) (median PFS: 3.00 months, 95\% CI 1.644.35 versus 8.00 months, $95 \%$ CI 5.80-10.19, respectively; $p=.000$ ) (Figure 1).

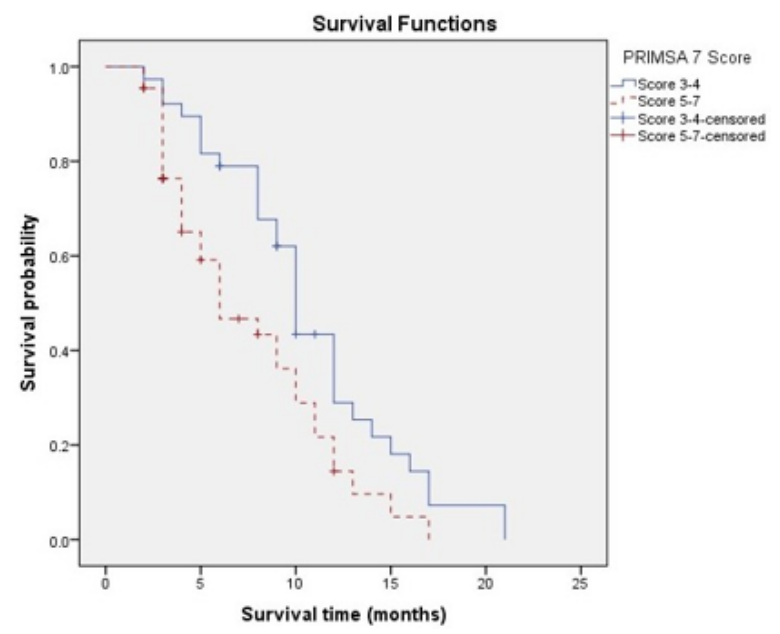

Figure 2: Kaplan-Meier plot overall survival according to PRISMA7 score.
Also, Overall Survival (OS) was markedly reduced in moderately to severely frail patients when compared to mildly frail patients (median OS: 6.00 months, 95\% CI 2.87-9.12 for patients with PRISMA7 score 3-4 versus 10.00 months, 95\% CI 9.10-10.89 for those with PRISMA7 score 5-7; $\mathrm{p}=.012$ ) (Figure 2).

\section{Conclusion}

In our opinion, PRIMSA7 score should be used instead of Performance Status (PS) for making a decision of active treatment in frail patients with pancreatic cancer. This is because of, firstly, the difficulty of assessing PS in these patients, secondly, the highly significant results regarding the prognostic value of PRISMA7 score in those patients.

\section{References}

1. Tas F, Sen F, Keskin S, Kilic L, Yildiz I (2013) Prognostic factors in metastatic pancreatic cancer: older patients are associated with reduced overall survival. Mol Clin Oncol 1(4): 788-792.

2. Macchini M, Chiaravalli M, Zanon S, Peretti U, Mazza E, et al. (2009) Chemotherapy in elderly patients with pancreatic cancer: Efficacy, feasibility and future perspectives. Cancer Treat Rev 72:1-6.

3. Luo G, Guo M, Liu Z, Xiao Z, Jin K, et al. (2015) Blood neutrophil-lymphocyte ratio predicts survival in patients with advanced pancreatic cancer treated with chemotherapy. Ann Surg Oncol 22(2): 670-676.

4. Lee SH, Jo JH, Kim YJ, Lee HS, Chung MJ, et al. (2019) Plasma chromogranin $\mathrm{A}$ as a prognostic marker in pancreatic ductal adenocarcinoma. Pancreas 48(5): 662-669.

5. Ngo-Huang A, Holmes HM, des Bordes JKA, Parker NH, Fogelman D, et al. (2019) Association between frailty syndrome and survival in patients with pancreatic adenocarcinoma. Cancer Med 8(6): 2867-2876.

6. Bray F, Ferlay J, Soerjomataram I, Siegel RL, Torre LA, et al. (2018) Global cancer statistics 2018: GLOBOCAN estimates of incidence and mortality worldwide for 36 cancers in 185 countries. CA Cancer J Clin 68(6): 394424 .

7. Altekruse SF, Kosary CL, Krapcho M, Neyman N, Aminou R, et al. (2010) SEER cancer statistics review, 1975-2007. National Cancer Institute.

8. Hentic O, Dreyer C, Rebours V, Zappa M, Levy P, et al. (2011) Gemcitabine in elderly patients with advanced pancreatic cancer. World J Gastroenterol 17(30): 3497-3502.

9. Aldoss IT, Tashi T, Gonsalves W, Ranin K, Xiang F, et al. (2011) Role of chemotherapy in the very elderly patients with metastatic pancreatic cancer - a Veterans Affairs Cancer Registry analysis. J Geriatr Oncol 2(3): 209-214.

10. Stewart BW (2014) World Cancer Report. Chapter 5.7, World Health Organization, IARC Publications, France.

11. Marechal R, Demols A, Gay F, Viviane M, Marianna A, et al. (2008) Tolerance and efficacy of gemcitabine and gemcitabine-based regimens in elderly patients with advanced pancreatic cancer. Pancreas 36(3): e16-e21.

12. Puts MT, Sattar S, McWatters K, Lee K, Kulik M, et al. (2017) Chemotherapy treatment decision-making experiences of older adults with cancer, their family members, oncologists and family physicians: A mixed methods study. Support Care Cancer 25(3): 879-886.

13. Bellera CA, Artaud F, Rainfray M, Soubeyran PL, Mathoulin-Pélissier S (2017) Modeling individual and relative accuracy of screening tools in geriatric oncology. Ann Oncol 28(5): 1152-1157.

14. Extermann M, Aapro M, Bernabei R, Cohen HJ, Droz JP, et al. (2005) Use of comprehensive geriatric assessment in older cancer patients: recommendations from the task force on CGA of the International Society of 
Geriatric Oncology (SIOG). Crit Rev Oncol Hematol 55(3): 241-252.

15. Dent E, Kowal P, Hoogendijk EO (2016) Frailty measurement in research and clinical practice: A review. Eur J Intern Med 31: 3-10.
16. Clegg A, Rogers L, Young J (2015) Diagnostic test accuracy of simple instruments for identifying frailty in community-dwelling older people: $\mathrm{A}$ systematic review. Age Ageing 44(1): 148-152. 